\title{
Evidence of capsaicin synthase activity of the Pun1-encoded protein and its role as a determinant of capsaicinoid accumulation in pepper
}

\author{
Kana Ogawa ${ }^{1}$, Katsunori Murota ${ }^{1}$, Hanako Shimura ${ }^{1}$, Misaki Furuya ${ }^{1}$, Yasuko Togawa $^{1}$, Takeshi Matsumura ${ }^{2}$
} and Chikara Masuta ${ }^{1 *}$

\begin{abstract}
Background: Capsaicinoids, including capsaicin and its analogs, are responsible for the pungency of pepper (Capsicum species) fruits. Even though capsaicin is familiar and used daily by humans, the genes involved in the capsaicin biosynthesis pathway have not been well characterized. The putative aminotransferase (pAMT) and Pungent gene 1 (Pun1) proteins are believed to catalyze the second to last and the last steps in the pathway, respectively, making the Pun1 protein the putative capsaicin synthase. However, there is no direct evidence that Pun1 has capsaicin synthase activity.

Results: To verify that the Pun1 protein actually plays a role in capsaicin production, we generated anti-Pun1 antibodies against an Escherichia coli-synthesized Pun1 protein and used them to antagonize endogenous Pun1 activity. To confirm the anti-Pun1 antibodies' specificity, we targeted Pun1 mRNA using virus-induced gene silencing. In the Pun1-down-regulated placental tissues, the accumulated levels of the Pun1 protein, which was identified on a western blot using the anti-Pun1 antibodies, were reduced, and simultaneously, capsaicin accumulations were reduced in the same tissues. In the de novo capsaicin synthesis in vitro cell-free assay, which uses protoplasts isolated from placental tissues, capsaicin synthesis was inhibited by the addition of anti-Pun1 antibodies. We next analyzed the expression profiles of PAMT and Pun 1 in various pepper cultivars and found that high levels of capsaicin accumulation always accompanied high expression levels of both PAMT and Pun1, indicating that both genes are important for capsaicin synthesis. However, comparisons of the accumulated levels of vanillylamine (a precursor of capsaicin) and capsaicin between pungent and nonpungent cultivars revealed that vanillylamine levels in the pungent cultivars were very low, probably owing to its rapid conversion to capsaicin by Pun 1 soon after synthesis, and that in nonpungent cultivars, vanillylamine accumulated to quite high levels owing to the lack of Pun1.
\end{abstract}

Conclusions: Using a newly developed protoplast-based assay for de novo capsaicin synthesis and the anti-Pun 1 antibodies, we successfully demonstrated that the Pun 1 gene and its gene product are involved in capsaicin synthesis. The analysis of the vanillylamine accumulation relative to that of capsaicin indicated that Pun1 was the primary determinant of their accumulation levels.

Keywords: Capsicum, pAMT, Pun1, Protoplast assay, Pungency, Vanillylamine, Virus-induced gene silencing

\footnotetext{
* Correspondence: masuta@res.agr.hokudai.ac.jp

${ }^{1}$ Graduate School of Agriculture, Hokkaido University, Sapporo 060-8589,

Japan

Full list of author information is available at the end of the article
} 


\section{Background}

Capsaicin is the compound responsible for the pungency of peppers [1]. Capsaicin and its analogs are called capsaicinoids, and the fundamental structure of most capsaicinoids is a branched-chain fatty acid amide of vanillylamine. Capsaicinoids, produced only by species of the genus Capsicum, are specifically synthesized in placental tissues, mostly between 20 and 30 days after flowering (daf) in the pepper fruits of pungent cultivars [2]. Capsaicinoids are used in the human diet for their distinct pungent taste and bioactivities, such as thermogenesis [3,4] and the suppression of fat accumulation [5]. In addition, they are used for pharmaceutical purposes because they have potential bioactivities that are ascribed to antioxidants [6] and anti-cancer agents [7]. Although pungency in peppers is a desirable trait, nonpungent cultivars also have been developed as vegetables. Nonpungent cultivars have been shown either to accumulate very few capsaicinoids or to accumulate capsinoids, such as capsiate, which are capsaicinoid analogs (branched-chain fatty acid ester of vanillyl alcohol) that lack the stimuli of the capsaicinoids [8].

Two pathways are involved in capsaicin biosynthesis: (1) the phenylpropanoid pathway derived from phenylalanine leading to vanillylamine; and (2) the branchedchain fatty acid pathway derived from valine leading to 8-methyl-6-nonenoyl-CoA [9-11]. The condensation reaction of vanillylamine with 8-methyl-6-nonenoyl-CoA is thought to be catalyzed by a coenzyme A-dependent acyltransferase. Although the genes involved in capsaicinoid synthesis have been extensively studied, the gene responsible for the acylation of vanillylamine remained unknown until Kim et al. [12] reported the SB2-66 clone as a possible candidate. Stewart et al. [13] finally showed that SB2-66 was the putative acyltransferase involved in capsaicinoid production and encoded by AT3 gene, namely Pungent gene 1 (Pun1) as capsaicin synthase (CS). The expression profile of Pun1 correlates with pepper pungency and the deletion or down-regulation of the Pun1 gene results in a decreased accumulation of capsaicinoids. In addition to capsaicinoids, Pun 1 has been reported to control capsinoid synthesis in nonpungent pepper cultivars $[14,15]$.

Another important step in capsaicin biosynthesis is the conversion of vanillin to vanillylamine, and a putative aminotransferase (pAMT) has been proposed to be the enzyme responsible for vanillin's transamination. The full-length cDNA clone of $p A M T$ was identified from a cDNA library by differential display [16]. Abraham-Juárez et al. [17] showed reduced capsaicinoid levels using virus-induced gene silencing (VIGS) against $P A M T$, providing evidence that the putative $p A M T$ gene was involved in capsaicinoid biosynthesis. In addition, Lang et al. [18] reported that the capsaicinoids were poorly synthesized in a spontaneous mutant, cultivar 'CH-19 Sweet', and that this phenotype was due to the loss-of-function of the $p A M T$ gene in the particular mutant. Using this mutant, Tanaka et al. [19] analyzed the defective $p A M T$ gene in detail and found that a single amino acid substitution was responsible for the capsaicin deficiency.

Very recently, pAMT was demonstrated, using an Escherichia coli-expressed recombinant enzyme, to act as a vanillin aminotransferase [20]. However, Pun1 is considered a putative gene because its encoded enzyme has not been biochemically analyzed, even though functional genomics studies indicate that Pun1 is responsible for the acylation of vanillylamine and vanillyl alcohol in Capsicum sp. [13-15]. In the present study, we used protoplasts and anti-Pun1 antibodies to verify that the Pun1 gene is actually involved in the crucial step of capsaicin biosynthesis. In addition, we investigated the expression profiles for two important genes, $p A M T$ and Pun1, in capsaicin biosynthesis and discussed the roles of pAMT and Pun1 in various pepper cultivars that differ in pungency.

\section{Results}

\section{Preparation of anti-Pun1 antibodies against the Pun1 protein to antagonize endogenous Pun 1 activity in an in vitro assay}

To verify that the Pun1 protein (syn. AT3) is the actual acyltransferase for capsaicin production, we first developed an in vitro assay system for capsaicin synthesis using recombinant Pun1 proteins, which are produced from the cDNA clone of Pun1 inserted into an E. coli expression vector. However, in preliminary experiments, we were not able to use recombinant Pun1 proteins in the enzymatic activity assay because they were mostly insoluble when expressed in E. coli, as reported previously [13]. Alternatively, we produced anti-Pun1 antibodies using the E. coli-synthesized Pun1 protein and tried to use them as antagonists of endogenous Pun1 activity in the in vitro assay system. To test the specificity of the created antibodies for the Pun1 protein, we first conducted a western blot analysis using total proteins from a nonpungent bell pepper, which is defective in the Pun1 gene, as a true negative control. As shown in Figure 1, we detected a very strong band in proteins from a pungent cultivar ('Chosen') at the expected size of $52 \mathrm{kDa}$, whereas there was no $52 \mathrm{kDa}$-signal in the bell pepper. We tested whether the antibodies crossreact with another acyltransferase, hydroxycinnamoyl transferase (HCT), which is also listed as a candidate enzyme in the capsaicinoid synthesis pathway [21,22]. The cDNA of the pepper HCT (EU616565) with the FLAG tag sequence at the $3^{\prime}$ end was polymerase chain reaction (PCR)-amplified and then synthesized as the HCT-FLAG protein from in vitro-transcribed HCT-FLAG 


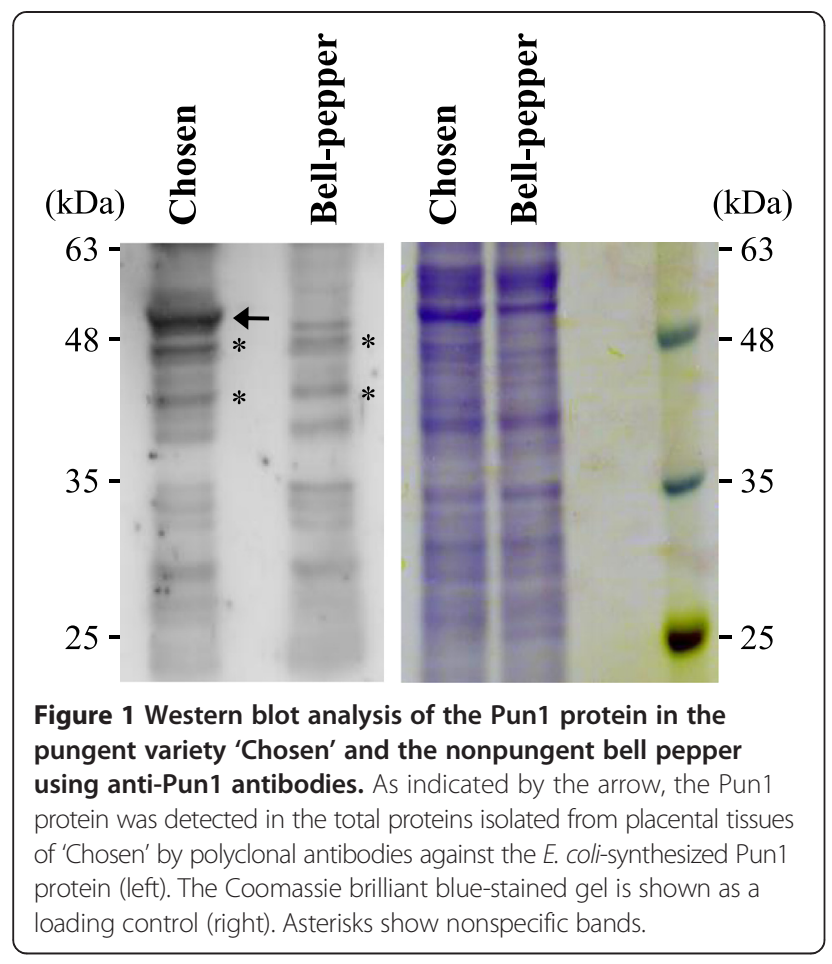

mRNA in a wheat germ system. The HCT-FLAG protein was clearly detected by an anti-FLAG antibody, but not by the anti-Pun1 antibodies, suggesting that the anti-Pun1 antibodies did not cross-react with the pepper HCT (Additional file 1: Figure S1). To further confirm the antibody specificity, we used VIGS to specifically reduce the expression level of Pun 1 mRNA and analyzed the capsaicinoid accumulation in the virus-infected pepper placenta. A 95-nt sequence of Pun1 was inserted in the Cucumber mosaic virus (CMV) vector (CMV-Yd:CS95) to induce RNA silencing against Pun1 mRNA. The pepper fruits infected with the virus vector containing the Pun1 insert showed no apparent differences from those infected with the empty vector. Compared with healthy fruits, the virus-infected fruits were a little smaller but no great differences were observed. The quantitative reverse-transcription (RT)-PCR analysis confirmed that the Pun1 mRNA levels were greatly reduced in the CMV-Yd:CS95-infected placental tissues (Figure 2A) in which the capsaicin accumulation was significantly reduced (Additional file 2: Figure S2). We next examined the Pun1 protein accumulation levels using a western blot analysis with the anti-Pun1 antibodies. As shown in Figure 2B, the Pun1 accumulation levels were indeed reduced in the CMV-Yd:CS95-infected placental tissues compared with those of the healthy and CMV-Ydinfected control tissues. These results suggest that the Pun1 expression was specifically reduced by VIGS, and that the anti-Pun 1 antibodies could detect the reduced levels of the Pun 1 protein in a western blot. Consistent with those results, the capsaicinoid content in the same placental tissues infected with CMV-Yd:CS95 was also reduced to between $\sim 25 \%$ and $33 \%$ of the control content (Figure 2C). The reduced level of the Pun1 protein detected by our antibodies reasonably reflected the reduced levels of capsaicinoid, and thus, we concluded that the specificity of the antibodies for the Pun 1 protein was satisfactory.

\section{Evidence that the Pun 1-encoded enzyme can catalyze capsaicin synthesis}

As described above, we produced anti-Pun1 antibodies using the E. coli-synthesized Pun1 protein and used the antibodies as antagonists of Pun1 activity. To develop an in vitro assay system for capsaicin synthesis, we first prepared cell-free crude enzyme extracts from the placental tissue of a pungent pepper and then added vanillylamine. However, we were not able to detect any de novo-synthesized capsaicinoid, probably owing to an unknown inhibitor(s). We therefore isolated protoplasts from placental tissues and used them for the assay (Figure 3A). In this assay, CS would be released from the protoplasts soon after the cells are broken, which occurs when vanillylamine is added. In the protoplast-based assay, we were able to detect de novo-synthesized capsaicin (Additional file 3: Figure S3). Capsaicinoid synthesis requires 8-methyl-6nonenoyl-CoA, as well as vanillylamine, as substrates, but 8-methyl-6-nonenoyl-CoA is not commercially available. In our protoplast-based assay, 8-methyl-6-nonenoyl-CoA was provided from the placental protoplasts, leading to capsaicin synthesis. Using this assay system, we analyzed whether the addition of the anti-Pun1 antibodies to the reaction would prevent de novo capsaicinoid synthesis. As we expected, the addition of anti-Pun1 antibodies significantly reduced capsaicin synthesis to less than half of the control without anti-Pun1 antibodies (Figure 3B, Additional file 3: Figure S3), suggesting that the Pun1 protein plays an essential role in capsaicin synthesis.

\section{Comparisons of expression levels of pAMT and Pun 1 between pungent and nonpungent cultivars}

To understand the roles of pAMT and Pun1 in capsaicinoid accumulation, we analyzed the gene expression levels of $p A M T$ and Pun1 in various pepper cultivars that differ in pungency. We extracted RNA from the placental tissues 25 daf and analyzed the expression levels of $p A M T$ using quantitative RT-PCR (Figure 4A). The expression levels of $p A M T$ were significantly higher in the pungent varieties than in the mildly pungent and nonpungent varieties, while $p A M T$ transcripts were barely detectable in the nonpungent varieties, suggesting that the more pungent the variety, the higher the $p A M T$ expression. We next measured the Pun1 transcript levels using the same RNA used for the analyses of $p A M T$. As 
A

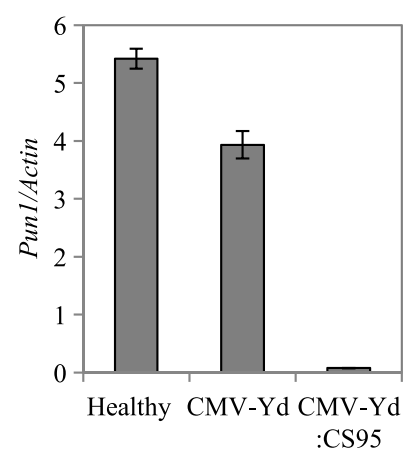

B

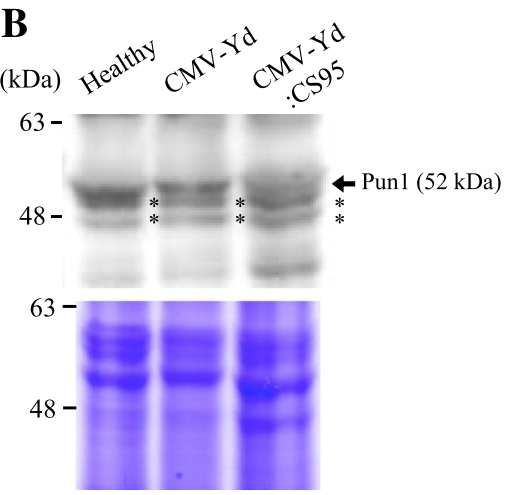

C

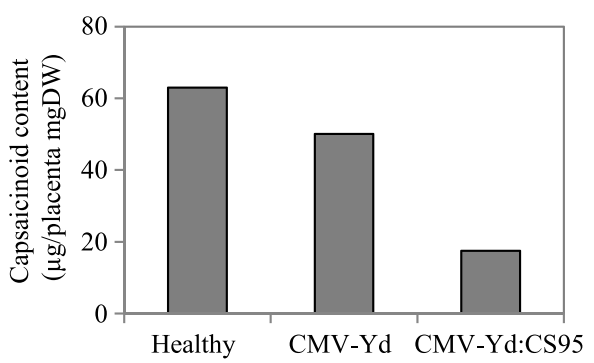

Figure 2 Effect of virus-induced gene silencing against the Pun 1 gene in pepper placental tissues. (A) Pun $1 \mathrm{mRNA}$ accumulation levels in placental tissues infected with CMV-Yd:CS95. CMV-Yd and CMV-Yd:CS95 are the empty vector and the vector containing a 95-nt sequence of the Pun 1 gene, respectively. The Pun 1 mRNA levels are shown relative to the Actin mRNA level in placental tissues that were all harvested at 20 to 25 days after flowering. Values are means \pm SD obtained from three replicates. (B) Western blot analysis of the Pun1 protein in CMV-Yd:CS95-infected placental tissues. The Coomassie brilliant blue -stained gel containing the same proteins as in the blot is shown as a loading control. Asterisks indicate nonspecific bands. (C) Capsaicinoid accumulation levels in CMV-Yd:CS95-infected placental tissues evaluated by HPLC. The same tissues were used for HPLC analysis as those used for western blotting.

shown in Figure 4B, the Pun1 transcript levels were relatively high in the pungent cultivars, but very low in the nonpungent cultivars. Just like for $p A M T$, the more pungent the cultivar, the higher the Pun1 expression. High-performance liquid chromatography (HPLC) analyses showed that the pungent cultivars produced higher levels of capsaicin (Figure 4C). Thus, the accumulation levels of capsaicinoids seem to correlate with the expression levels of Pun1 and $p A M T$.

When we examined the expression levels of $p A M T$ and Pun1 over time using quantitative RT-PCR (Figure 5A), as expected, the expression levels of $p A M T$ and Pun1 were very high in the pungent cultivar 'Chosen' but barely present in the nonpungent cultivar 'Fushimi'. It is noteworthy that Pun1 mRNA in 'Chosen' decreased after 25 daf when capsaicin reached its maximum level in the placenta (Figure $5 \mathrm{~B}$ ), whereas $p A M T$ mRNA continued to increase even at 35 daf.

\section{Comparison of vanillylamine contents between pungent and nonpungent varieties}

To confirm that Pun1, rather than $p A M T$, is the primary determinant of capsaicinoid accumulation during capsaicin synthesis, we compared vanillylamine levels between pungent and nonpungent cultivars. The HPLC analyses showed significantly greater levels of vanillylamine in the placental tissues of nonpungent cultivars at 25 daf (Figure 6A). This result was unexpected because we had initially thought that the vanillylamine contents would be relatively low owing to the low pAMT expression in nonpungent cultivars. When we analyzed vanillylamine levels in immature placental tissues at 11 daf, vanillylamine was relatively abundant in the pungent cultivar compared with the nonpungent cultivar (Figure 6B). Thus, we assumed that most of the vanillylamine was efficiently converted to capsaicinoids by Pun1, which was present at high levels soon after vanillylamine synthesis in pungent cultivars at 25 daf. However, the very low levels of Pun1 present in nonpungent cultivars were probably not sufficient to produce capsaicin despite the high accumulation of vanillylamine. However, low levels of pAMT were still capable of converting vanillin to vanillylamine in nonpungent cultivars. Thus, we hypothesize that Pun1 is a more critical regulator of capsaicin synthesis than pAMT. 


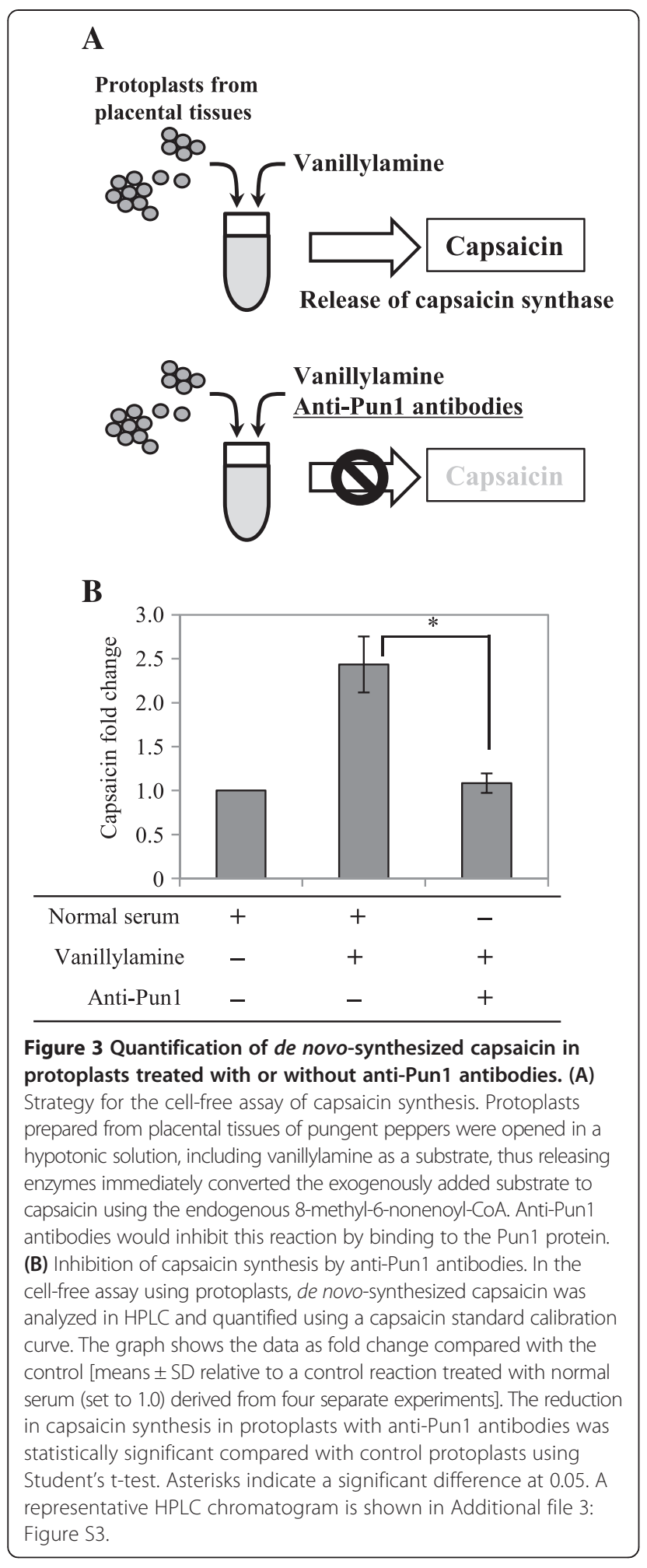

\section{Discussion}

Previous knockdown experiments of the $p A M T$ and Pun 1 genes by VIGS demonstrated that the down-regulation of these genes resulted in reduced capsaicinoid contents in fruits $[13,17]$, indicating that both genes are responsible for the last steps in capsaicinoid synthesis. In this study, we obtained a similar result with the VIGS targeting of the Pun1 gene using the CMV vector. The whole-genome sequencing of pepper revealed that there were three copies of the AT3 (Pun1) gene [23]. According to their genome structures and fruit-specific gene expression levels, AT3D1 and AT3-D2 may be functional in capsaicinoid biosynthesis. Because they are very similar at the $95 \mathrm{nt}$ long target sequence for VIGS, we assumed that both genes were simultaneously down-regulated by VIGS. We then compared the expression levels of the pAMT and Pun1 genes and the capsaicin accumulation levels between pungent and nonpungent cultivars. The quantitative RT-PCR analyses indicated that the capsaicinoid levels correlated well with the expression levels of both pAMT and Pun1, with expression of the two genes in the pungent cultivars being 50- to 100-fold higher than in the nonpungent cultivars. These results strongly suggest that capsaicinoid levels (pepper pungency) are primarily determined by the expression levels of both $p A M T$ and Pun1, which are exclusive to the pungent cultivars. Presently, we cannot completely eliminate the possibility that the enzymatic activities of pAMT and/or Pun1 differ between pungent and nonpungent cultivars. However, it is unlikely that the enzymatic activities of pAMT and/or Pun1 are the determinants of pepper pungency over their transcriptional levels because their mRNA levels in the nonpungent cultivars ranged from extremely low to undetectable in our experiments. Thus, the enzymes per se do not accumulate in the placenta, regardless of their activity levels. The accumulation of the AT3 (Pun1) protein is reported to be closely correlated with the level of AT3 (Pun1) transcripts in the pungent cultivar 'Thai Hot' [13]. Additionally, Pun1 is highly expressed in the pungent cultivar $C$. chinense 'Habanero' at the transcript and protein levels, but was hardly detectable in the nonpungent cultivars $C$. аnnuum 'Maor' and $C$. chinense NMCA30036 [24]. These reports indicate that capsaicinoid biosynthesis may be regulated primarily at the transcription level. Although the $P A M T$ expression level in the nonpungent cultivar C. апnиит 'CH-19 Sweet' was reported to be comparable with that in the pungent cultivars, this would be an exceptional case because 'CH-19 Sweet' accumulates capsinoids instead of capsaicinoids [18].

The transamination of vanillin to vanillylamine and the acylation of vanillylamine with a branched-chain fatty acid are critical steps in the biosynthesis of capsaicinoids $[10,11]$. The most plausible candidate genes for the acylation, Pun1, and the putative aminotransferase gene, $p A M T$, have been highly correlated with capsaicinoid accumulation in several genetic studies of mutant alleles that corresponded to either $P A M T$ or Pun 1 $[13,18,19]$. However, because the enzymatic activities of pAMT and/or Pun1 may differ between the pungent 


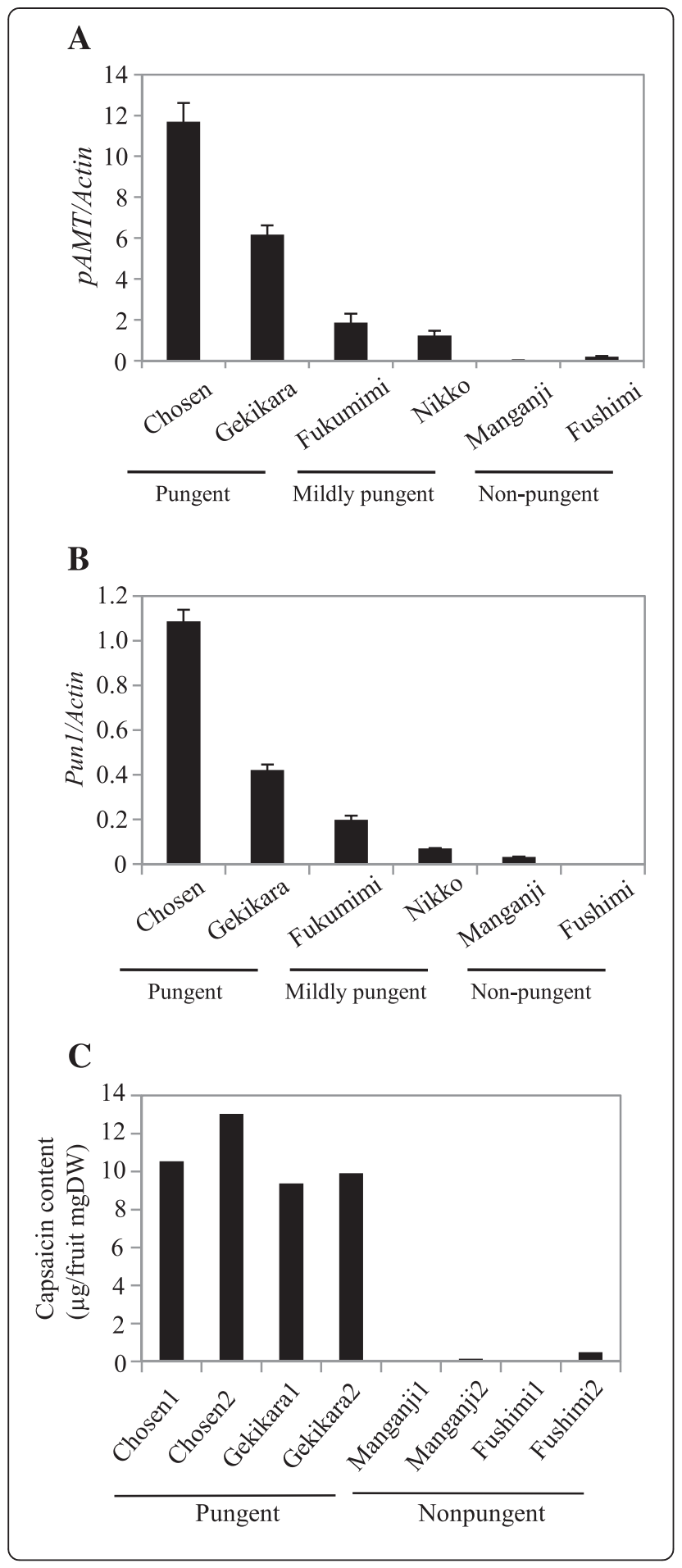

Figure 4 Relationships between the expression levels of $p A M T$ and Pun 1 genes, and the accumulation of capsaicin in different pepper cultivars. (A) PAMT expression in different pepper cultivars. RNA transcript levels for the pAMT gene at 25 days after flowering in placental tissues were determined using quantitative RT-PCR (mean $\pm \mathrm{SE} ; n=3$ ). 'Chosen' and 'Gekikara': pungent pepper cultivars; 'Nikko' and 'Fukumimi': mildly pungent pepper cultivars; 'Fushimi' and 'Manganji': nonpungent pepper cultivars. (B) Pun1 gene expression in different pepper cultivars. Pun1 transcript levels were determined using quantitative RT-PCR (means $\pm S E_{;} n=3$ ) with the same RNA as that used for the PAMT analyses. (C) Capsaicin levels in pungent and nonpungent pepper cultivars as determined by HPLC.

and nonpungent cultivars, we should be able to measure the enzymatic activities of the enzymes. Thus, biochemical verifications will still be necessary to determine whether these putative genes are the real players in the pathway. Recently, based on bioinformatics analyses, single nucleotide polymorphisms in the Pun1 locus were found to distinguish pungent from nonpungent cultivars $[25,26]$. This suggests that Pun1 is indeed the locus responsible for the qualitative effect on pungency in pepper cultivars and that the enzymatic activity of Pun1 may be categorized into two levels, which determine whether a cultivar will be pungent or not. Since Kim et al. [14] reported that cDNA clone SB2-66 is identical to the Pun1 gene, Pun1 has been suspected to be the actual CS, but there has been no cellfree system to synthesize capsaicin. Purified proteins with CS activity have suffered from contamination with endogenous capsaicinoids; therefore, researchers must use a radioactive substrate, which is not commercially available. Here, we overcame these technical difficulties by using protoplasts isolated from placental tissues of a pungent cultivar. This protoplast-based assay is so simple and easy that comparative experiments assessing Pun1 activities among pepper cultivars are now possible. In addition, this system can be used for other purposes, such as measuring other enzymatic activities and screening for capsaicin synthesis inhibitors.

Even though the transcript levels of both $p A M T$ and Pun1 were very low in the nonpungent cultivars compared with the pungent cultivars, the vanillylamine levels were five times higher in the nonpungent cultivars than in the pungent cultivars at 25 daf (Figure 6). Although these results seem to contradict each other, one possible explanation is that the vanillylamine synthesized in the pungent cultivars was quickly converted to capsaicin by the high Pun1 enzyme activity (Additional file 4: Figure S4). According to this hypothesis, which is based on an expression time course of $p A M T$ and Pun 1 relative to the vanillylamine accumulation levels, vanillylamine must be efficiently synthesized in nonpungent cultivars even when there is a very low $p A M T$ expression level at 25 daf. From this point of view, the nonpungent feature is substantially determined by the loss of function 


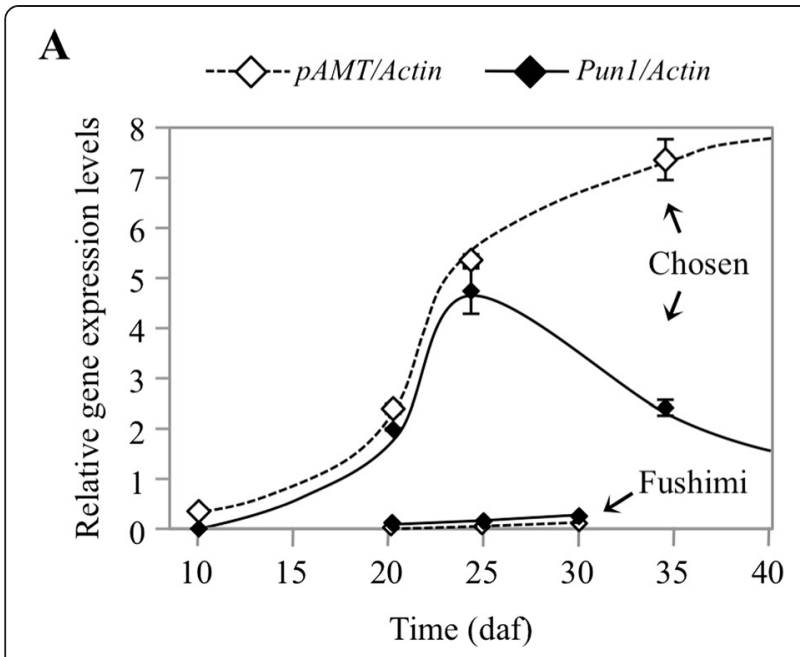

B

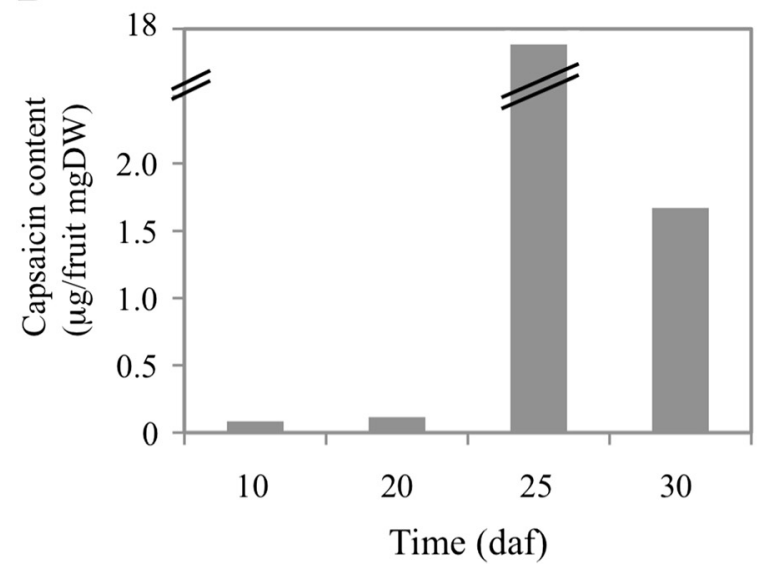

Figure 5 Gene expression levels of $p A M T$ and Pun 1 and capsaicin levels during fruit development. (A) Quantitative RT-PCR to determine RNA levels of PAMT and Pun1 in placental tissues (means \pm SE; $n=3$ ). Samples of 'Chosen' (pungent) were harvested at 10, 20, 25 and 35 days after flowering, and samples of 'Fushimi' (nonpungent) were harvested at 20, 25 and 30 days after flowering. (B) Capsaicin levels in the fruits of 'Chosen' (pungent) during fruit development as analyzed by HPLC.

of Pun1 (or very low Pun1 expression levels), and thus the nonpungent phenotype, with relatively high vanillylamine levels, was determined essentially by low expression levels of Pun1 but not of $p A M T$. Therefore, Pun1 would be the primary determinant of vanillylamine, as well as capsaicin, accumulation. A nonpungent cultivar C. annuum 'CH-19 Sweet' [18] was shown to accumulate less vanillylamine than pungent cultivars, but this case is exceptional because 'CH-19 Sweet' must have enough Pun1 activity to accumulate a high capsinoids (capsiate) content, unlike the nonpungent cultivars used in this study that showed very low Pun1 expression levels.

\section{Conclusions}

To verify that the Pun1 gene is actually involved in the final step of capsaicin biosynthesis, we raised antibodies

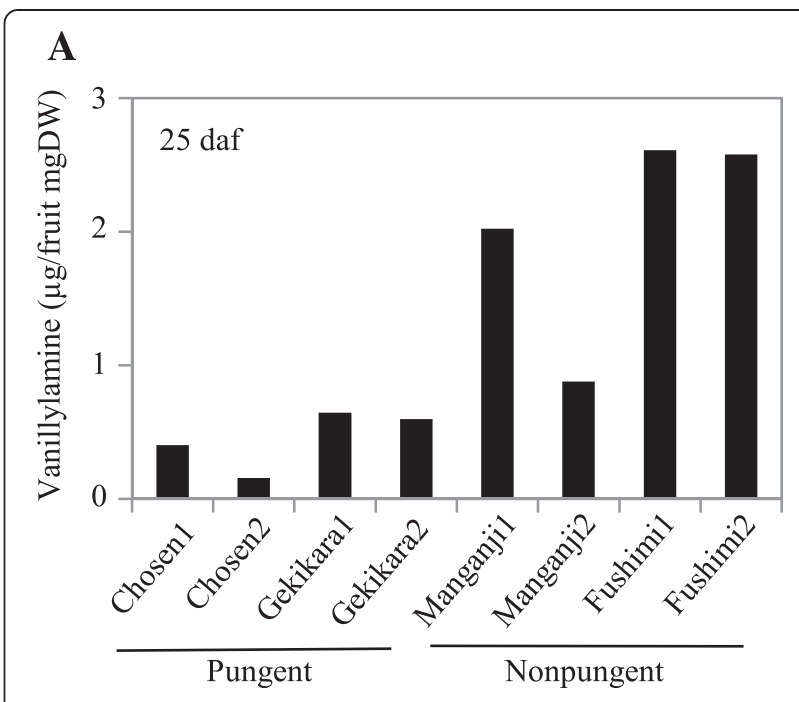

B

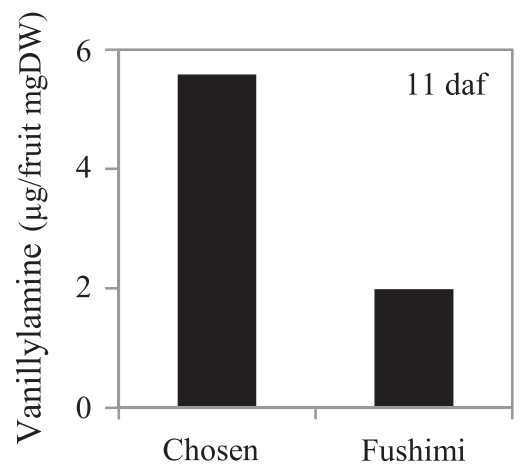

Figure 6 Vanillylamine levels in pungent and nonpungent pepper cultivars. (A) Vanillylamine levels at 25 days after flowering. (B) Vanillylamine levels at 11 days after flowering. Freeze-dried pepper fruits were ground, and vanillylamine was extracted with $0.1 \%$ acetic acid methanol and then analyzed by HPLC at $280 \mathrm{~nm}$. The $x$-axis in (A) shows individual fruits. Three fruits were used for (B).

against the $E$. coli-synthesized Pun1 protein and used them as antagonists of endogenous Pun1 activity. After confirmation of the antibodies' specificity, we developed an in vitro cell-free assay for de novo capsaicin synthesis using protoplasts from placental tissues. The addition of anti-Pun 1 antibodies significantly reduced de novo capsaicin synthesis in the protoplast assay, demonstrating that the Pun1-encoded protein played an essential role in capsaicin synthesis. Analyses of the accumulation of vanillylamine, a precursor of capsaicin, revealed that nonpungent cultivars could accumulate much higher vanillylamine levels than pungent cultivars. This observation indicates that Pun 1 is the primary determinant of vanillylamine and capsaicin accumulation and is more important than $p A M T$ in maintaining capsaicin-free fruit in most nonpungent cultivars. 


\section{Methods}

Plant materials

Capsicum annuum (L.) cultivars 'Chosen,' 'Gekikara' and 'Kaien' were used as the pungent cultivars, 'Nikko' and 'Fukumimi' as the mildly pungent, and 'Fushimi' and 'Manganji' as the nonpungent cultivars. C. annuum var. grossum (bell pepper) was also used as a capsaicinoid-free control pepper. Plants were cultured in an incubation room under $16 \mathrm{~h}$ of fluorescent light at $24^{\circ} \mathrm{C}$ and $50 \%$ relative humidity. Green fruits at $\sim 25$ daf were used for the experiments unless otherwise indicated.

\section{Cloning of the $P A M T$ and Pun 1 genes}

Total RNA was extracted from the placental tissues of pepper fruits using TRIZOL reagent (Ambion) according to the manufacturer's instructions. For cloning $p A M T$ and Pun1, total RNA extracted from 'Chosen' was used as a template for reverse transcription with AMV Reverse Transcriptase (NIPPON GENE). The full-length cDNAs for $p A M T$ and Pun1 were amplified by PCR with the primer set 5' $5^{\prime}$-ATGGCCAATATTACTAATG-3' and 5'-T TAATGCTTCTGAGAC-3' and the primer set $5^{\prime}$-ATG GCTTTTGCATTACCATC-3' and 5'-TTAATTAGGCA ATGAACTCAAGG-3', respectively. The pGEM-T Easy Vector System I (Promega) was used for the subcloning and subsequent sequencing of the PCR products. Sequencing was performed with the BECKMAN COULTER CEQ8800 system. We designed all primers based on published sequences of the $P A M T$ (AF085149) and Pun1 (AY819029) genes.

\section{Quantitative RT-PCR analysis}

cDNA was synthesized from DNase-treated RNA and amplified using KOD SYBR qPCR Mix (Toyobo) according to the manufacturer's instructions. mRNA levels were quantified by quantitative RT-PCR using the StepOne Real-Time PCR System (Applied Biosystems). The Actin gene was used as an internal control. Primer sets for each gene amplification were as follows: Actin, $5^{\prime}-\mathrm{G}$ GTTAAGGCTGGATTTGCTG-3' and 5' -ATGCATCC TTTTGACCCATC-3'; pAMT, 5'-GTAAGTTCCACTG GTGATCATG-3' and 5' ${ }^{\prime}$-TTACTGCTTCTGAGAC-3'; and Pun1, 5' ${ }^{\prime}$-AGGCATCATCAATGCTAC-3' and 5' ${ }^{\prime}$-A 'TGTTAGTTGCTTCTATGGAG-3'.

\section{Virus-induced gene silencing (VIGS) against Pun1}

The Cucumber mosaic virus (CMV) vector (CMV-A1 vector) had been used previously for VIGS experiments in pepper plants [27]. Here, we modified the CMV-A1 vector by changing an $R$ residue at position 46 into a $C$ residue in the $2 \mathrm{~b}$ protein $(2 \mathrm{~b})$, which weakened the RNA silencing suppressor activity of $2 \mathrm{~b}$ so that the viral symptoms became milder in the pepper. The modified CMV-A1 vector was designated CMV-Yd. We used a pungent pepper cultivar 'Kaien' for the VIGS experiments because CMVYd can systemically infect 'Kaien' and easily penetrate into placental tissues. A 95-bp fragment from the Pun1 gene was amplified by the primer pair $5^{\prime}$-CGCACGCGTG AAGGAAGTTGAGGTGGCATA-3' and 5' -CGAGGCC 'TGAGCAGTTTCCCTTCTCTCATTG-3' and inserted between the $S m a \mathrm{I}$ and $M l u \mathrm{I}$ sites in the CMV-Yd vector to create CMV-Yd:CS95.

\section{Extraction of vanillylamine and capsaicin}

Fruits were harvested, frozen and dried completely in a freeze-dryer (FDU-1200, EYELA) for 2 d. Dried fruits were then ground in a blender (Microsmash MS100, TOMY) at room temperature. For the vanillylamine extraction, $1 \mathrm{ml}$ of $0.1 \%$ acetic acid methanol was added to $100 \mathrm{mg}$ of dry fruit powder. For the capsaicin extraction, $0.1 \%$ acetic acid acetonitrile was added instead. After the samples were mixed, they were allowed to settle for $1 \mathrm{~h}$ at room temperature. The supernatant was passed through a $0.2 \mu \mathrm{m}$-pore membrane filter before being used in HPLC (JASCO LC-2000 system).

\section{HPLC analysis}

The samples were separated in a SHIM-PACK VP-ODS column $(150 \mathrm{~mm} \times 4.6 \mathrm{~mm}$; Shimazu). For vanillylamine, the eluent was a mixture of methanol/10 $\mathrm{mM}$ phosphate buffer $\mathrm{pH} 2.6(3: 2 \mathrm{v} / \mathrm{v})$, and the flow rate was $0.8 \mathrm{ml} / \mathrm{min}$. For capsaicin, the eluent was a mixture of acetonitrile/1\% acetic acid $(2: 1 \mathrm{v} / \mathrm{v})$, and the flow rate was $1 \mathrm{ml} / \mathrm{min}$. All eluates were monitored at $280 \mathrm{~nm}$ using a UV detector. External standards were prepared by dissolving commercial vanillylamine (Sigma-Aldrich) and capsaicin (SigmaAldrich) in methanol and acetonitrile, respectively.

\section{In vitro cell-free assay for capsaicin synthesis}

Protoplasts were isolated from fruits of the pungent cultivar 'Chosen' as previously described [28], except that cells were not shaken during the enzyme digestion and the reaction was stopped when the mass of cells (roughly 20-30 cells/mass) were released from the tissues. A 500- $\mu$ l sample of the protoplasts was transferred to a $1.5-\mathrm{ml}$ tube and centrifuged $\left(3 \mathrm{~min}, 100 \times g, 4^{\circ} \mathrm{C}\right)$ to remove the supernatant. Protoplasts were then resuspended in $200 \mu \mathrm{l}$ solution containing $1 \mathrm{mM}$ aqueous vanillylamine (Sigma-Aldrich). Protoplasts were ruptured to release cellular enzymes, including CS, by vortexing the mixture 4 times for $5 \mathrm{~s}$ each. After a 2-h incubation at room temperature, followed by centrifugation $\left(2 \mathrm{~min}, 6,000 \times g, 4^{\circ} \mathrm{C}\right)$ of the reaction mixture, the supernatant was collected and extracted with the ethyl acetate. The extract was dried overnight at room temperature in the dark, resuspended in $100 \mu \mathrm{l}$ of $0.1 \%$ acetic acid acetonitrile and then passed through a $0.2-\mu \mathrm{m}-$ pore membrane filter before HPLC. To inhibit CS activity, 
anti-Pun1 antibodies (described next) were added to the reaction mixture at a final dilution of 1:2000 before the in vitro capsaicin synthesis started.

\section{Expression of Pun1 in E. coli and production of anti-Pun1 antibodies}

The open-reading frame of the Pun1 gene was amplified with primer set 5'-CGGAATTCATGGCTTTTGCATT ACCATC-3' containing an EcoRI site and 5'-CGGGAT CCTAATTAGGCAATGAACTCAAGG-3' containing a $B a m H I$ site, and cloned between the EcoRI and BamHI sites of pMAL-c2x (New England Biolabs). The N-terminal maltose binding protein (MBP)-fused Pun1 recombinant protein was then expressed in E. coli (BL21) and affinitypurified using the pMAL Protein Fusion and Purification System (New England BioLabs). Anti-Pun1 antibodies, prepared by Frontier Institute Co. (Sapporo, Japan), were raised by immunizing a rabbit with the purified recombinant protein.

\section{Western blots}

Total proteins from pepper placentas, extracted as described by Masuta et al. [29], were separated on 10\% sodium dodecyl sulfate polyacrylamide gels (SDS-PAGE) and blotted on an Immobilon-P membrane (Millipore). The blots were then treated with primary antibodies raised against the E. coli-synthesized Pun1 protein, and subsequently with goat anti-rabbit IgG-alkaline phosphataseconjugate (Takara Bio) as a secondary antibody. Signals were visualized using CDP-Star (Roche) as a substrate.

\section{Additional files}

Additional file 1: Figure S1. Western blot analysis of the in vitro synthesized pepper hydroxycinnamoyl transferase $(\mathrm{HCT})$ protein using the anti-Pun1 antibodies. The CDNA clone of HCT was PCR-amplified and inserted in the pEU plasmid vector (CellFree Sciences, Japan). After in vitro transcription from the recombinant plasmid, HCT-fused to a C-terminal FLAG peptide (HCT-FLAG) was in vitro synthesized in the wheat germ cocktail (CellFree Sciences, Japan) according to the manufacturer's instructions. The $\mathrm{HCT}$ protein with a FLAG tag was then subjected to detection either by the anti-Pun1 antibodies (left) or by an anti-FLAG antibody (right). Note that anti-Pun1 antibodies did not react with the HCT protein. The arrow indicates the HCT protein.

Additional file 2: Figure S2. Accumulation levels of capsaicinoids in placental tissues infected with CMV-Yd:CS95. The graph shows the data as fold change compared with the healthy control (means \pm SD from three separate experiments using 3 to 5 pepper fruits for each treatment) The reduction in capsaicin accumulation in CMV-Yd:CS95 was statistically significant compared with the empty vector control (CMV-Yd) using Student's t-test. Asterisks indicate significance at 0.01 .

Additional file 3: Figure S3. HPLC chromatograms of de novo capsaicin synthesis products from the in vitro cell-free assay using protoplasts. The main peak (red) in each chart represents capsaicin. This experiment was repeated twice and produced the same results.

Additional file 4: Figure S4. Hypothesis to explain the accumulation of vanillylamine in nonpungent pepper cultivars. In pungent peppers, vanillylamine is quickly converted to capsaicin by highly active CS, while in nonpungent cultivars, vanillylamine is synthesized even with a very low pAMT activity level. Although vanillylamine is more abundant in nonpungent cultivars than in the pungent cultivars at 25 daf (dotted line), it will not be converted to capsaicin owing to the very low level of CS activity. This result is supported by the gene expression time-course of pAMT and Pun1 (Figure 5).

\section{Abbreviations}

CS: Capsaicin synthase; daf: Days after flowering; pAMT: Putative aminotransferase; Pun1: Pungent gene 1; HCT: Hydroxycinnamoyl transferase; VIGS: Virus-induced gene silencing.

\section{Competing interests}

The authors declare that they have no competing interests.

\section{Authors' contributions}

$\mathrm{CM}$ and $\mathrm{TM}$ conceived and designed the research. $\mathrm{KO}, \mathrm{KM}, \mathrm{MF}$ and $\mathrm{YT}$ conducted the experiments. $\mathrm{CM}$ and $\mathrm{KO}$ analyzed the data. $\mathrm{KO}, \mathrm{HS}$ and $\mathrm{CM}$ wrote the manuscript. All authors read and approved the manuscript. The first and second authors contributed equally.

\section{Acknowledgments}

We thank the Northern Advancement Center for Science and Technology (Noastech), Japan, for financial support. We also thank Dr. Kiyoshi Masuda for helping us operate the HPLC. We are grateful to Dr. Tatsuo Watanabe for providing us the capsaicin analog, octanoyl-CoA, which was used to practice the in vitro capsaicin synthesis.

\section{Author details}

${ }^{1}$ Graduate School of Agriculture, Hokkaido University, Sapporo 060-8589, Japan. ${ }^{2}$ Plant Molecular Technology Research Group, Research Institute of Bioproduction, National Institute of Advanced Industrial Science and Technology, Sapporo 062-8517, Japan.

Received: 15 January 2015 Accepted: 17 March 2015

Published online: 28 March 2015

\section{References}

1. Thresh CF. Capsaicin, the active principle of capsicum fruits. Pharmaceut J. 1876:315:21

2. Iwai K, Suzuki T, Fujiwake H. Formation and accumulation of pungent principle of hot pepper fruits, capsaicin and its analogues, in Capsicum annuum var. annuum cv. Karayatsubusa at different growth stage after flowering. Agric Biol Chem. 1986;43:2493-8.

3. Kawada T, Sakabe S, Aoki N, Watanabe T, Higata K, Iwai K, et al. Intake of sweeteners and pungent ingredients increases the thermogenin content in brown adipose tissue of rat. J Agric Food Chem. 1991;39:651-4.

4. Westerterp-Plantenga M, Diepvens $\mathrm{K}$, Joosen AMCP, Bérubé-Parent $\mathrm{S}$, Tremblay A. Metabolic effects of spices, teas, and caffeine. Physiol Behav. 2006:89:85-91.

5. Kawada T, Hagiwara K, Iwai K. Effects of capsaicin on lipid metabolism in rats fed a high fat diet. J Nutr. 1986;116:1272-8.

6. Srinivasan K. Antioxidant potential of spices and their active constituents. Cit Rev Food Sci Nutr. 2014;54:352-72.

7. Wutka A, Palagani V, Barat S, Chen X, Khatib ME, Gotze J, et al. Capsaicin treatment attenuates cholangiocarcinoma carcinogenesis. PLOS ONE. 2014;9:e95695.

8. Kobata K, Todo T, Yazawa S, Iwai K, Watanabe T. Novel capsaicinoid-like substances, capsiate and dihydrocapsiate, from the fruits of a nonpungent cultivar, CH-19 Sweet, of pepper (Capsicum annuum L.). J Agric Food Chem. 1998:46:1695-7.

9. Aza-Gonzalez C, Nunez-Palenuis HG, Ochoa-Alejo N. Molecular biology of capsaicinoids biosynthesis in chili pepper (Capsicum spp.). Plant Cell Rep. 2011:30:695-706.

10. Bennett DJ, Kirby GW. Constitution and biosynthesis of capsaicin. J Chem Soc. 1968:C:442-6.

11. Leete $\mathrm{E}$, Louden M. Biosynthesis of capsaicin and dihydrocapsaicin in Capsicum frutescens. J Am Chem Soc. 1968;90:6837-41.

12. Kim M, Kim S, Kim S, Kim BD. Isolation of CDNA clones differentially accumulated in the placenta of pungent pepper by suppression subtractive hybridization. Mol Cells. 2001;11:213-9. 
13. Stewart C, Kang BC, Liu K, Mazourek M, Moore SL, Yoo EY, et al. The Pun1 gene for pungency in pepper encodes a putative acyltransferase. Plant $\mathrm{J}$. 2005; $42: 675-88$

14. Han K, Jeong HJ, Sung J, Keum YS, Cho MC, Kim JH, et al. Biosynthesis of capsinoid is controlled by the Pun1 locus in pepper. Mol Breeding. 2013;31:537-48

15. Kobata K, Sugiwara M, Miura M, Yazawa S, Watanabe T. Potent production of capsaicinoids and capsinoids by Capsicum peppers. J Agri Food Chem. 2013;61:11127-32

16. Curry J, Aluru M, Mendoza M, Nevarez J, Melendre M, O'Connell MA. Transcripts for possible capsaicinoid biosynthetic genes are differentially accumulated in pungent and non-pungent Capsicum spp. Plant Sci. 1999;148:47-57.

17. Abraham-Juarez MR, Rocha-Granados MC, Lopez MG, Rivera-Bustamante RF, Neftali OA. Virus-induced silencing of Comt, pAMT, Kas genes results in a reduction of capsaicinoid accumulation in chili pepper fruits. Planta. 2008:227:681-95

18. Lang Y, Kisaka H, Sugiyama R, Nomura K, Morita A, Watanabe T, et al. Functional loss of pAMT results in biosynthesis of capsinoids, capsaicinoid analogs, in Capsicum annuum cv. CH-19 sweet. Plant J. 2009;59:953-61.

19. Tanaka Y, Hosokawa M, Miwa T, Watanabe T, Yazawa S. Newly mutated putative-aminotransferase in nonpungent pepper (Capsicum annuum) results in biosynthesis of capsinoids, capsaicinoid analogues. J Agri Food Chem. 2010:58:1761-7.

20. Weber N, Ismail A, Gorwa-Grauslund M, Carlquist M. Biocatalytic potential of vanillin aminotransferase from Capsicum chinense. BMC Biotechnol. 2014;14:25.

21. Hoffmann L, Besseau S, Geoffroy P, Ritzenthaler C, Meyer D, Lapierre C, et al. Silencing of hydroxycinnamoyl-coenzyme A shikimate/quinate hydroxycinnamoyltransferase affects phenylpropanoid biosynthesis. Plant Cell. 2004;16:1446-65.

22. Mazourek M, Pujar A, Borovsky Y, Paran I, Mueller L, Jahn MM. A dynamic interface for capsaicinoid systems biology. Plant Physiol. 2009;150:1806-21.

23. Qin C, Yu C, Shen Y, Fang X, Chen L, Min J, et al. Whole-genome sequencing of cultivated and wild peppers provides insights into Capsicum domestication and specialization. Proc Natl Acad Sci USA. 2014;111:5135-40.

24. Stewart C, Mazourek M, Stellari GM, O'Connell M, Jahn M. Genetic control of pungency in C. chinense via the Pun1 locus. J Exp Bot. 2007:58:979-91.

25. Reddy UK, Almeida A, Abburi V, Alaparthi SB, Unselt D, Hankins G, et al. Identification of gene-specific polymorphisms and association with capsaicin pathway metabolites in Capsicum annuum L. collections. PLoS ONE. 2014;9:e86393.

26. Hill TA, Ashrafi H, Reyes-Chin-Wo S, Yao J, Stoffel K, Truco MJ, et al. Characterization of Capsicum annuum genetic diversity and population structure based on parallel polymorphism discovery with a $30 \mathrm{~K}$ unigene pepper genechip. PLoS ONE. 2013;8:e56200.

27. Hong JS, Rhee SJ, Kim EJ, Kim TS, Ryu KH, Masuta C, et al. Application of a reassortant Cucumber mosaic virus vector for gene silencing in tomato and chili pepper plants. Plant Pathol J. 2012;28:81-6.

28. Pitzschke A, Persak H. Poinsettia protoplasts - a simple, robust and efficient system for transient gene expression studies. Plant Methods. 2012;8:14.

29. Masuta C, Tanaka H, Uehara K, Kuwata S, Koiwai A, Noma M. Broad resistance to plant viruses in transgenic plants conferred by antisense inhibition of a host gene essential in S-adenosylmethionine-dependent transmethylation reactions. Proc Natl Acad Sci USA. 1995;92:6117-21.

\section{Submit your next manuscript to BioMed Central and take full advantage of:}

- Convenient online submission

- Thorough peer review

- No space constraints or color figure charges

- Immediate publication on acceptance

- Inclusion in PubMed, CAS, Scopus and Google Scholar

- Research which is freely available for redistribution

Submit your manuscript at www.biomedcentral.com/submit 